\title{
Computation of Lyapunov Functions for Discrete-Time Systems using the Yoshizawa Construction
}

\author{
Huijuan $\mathrm{Li}^{*}$, Sigurður Hafstein ${ }^{\dagger}$, Christopher M. Kellett ${ }^{\ddagger}$
}

\begin{abstract}
In this paper, we present a new approach for computing Lyapunov functions for nonlinear discrete-time systems with an asymptotically stable equilibrium at the origin. The proposed method constructs a continuous piecewise affine (CPA) function on a compact subset of the state space containing the origin, given a suitable triangulation or partition of the compact set and values at the vertices of the triangulation. Here, the vertex values are fixed using a function from a classical converse Lyapunov theorem originally due to Yoshizawa. Several numerical examples are presented to illustrate the proposed approach.
\end{abstract}

\section{INTRODUCTION}

The Lyapunov function is among the most useful tools for stability analysis of dynamic systems since it allows one to conclude (asymptotic) stability of an equilibrium without knowledge of the explicit solution of the dynamic system. This utility has motivated the search for Lyapunov functions for dynamic systems for many years. In the present article, we focus on computing Lyapunov functions for discrete-time dynamic systems. Such systems are widely used to study practical phenomena in many fields such as engineering, finance, and biology.

Several methods have been proposed for computing Lyapunov functions for discrete-time dynamic systems. For instance, collocation methods were presented in [4] and [5], graph algorithms are used to compute complete Lyapunov functions in [3] and [11], and the continuous piecewise affine (CPA) method was extended to discrete-time systems in [7].

The CPA method is of particular interest since it delivers a true Lyapunov function for discrete-time dynamic systems on a compact subset of the state space. This method relies on a partitioning of the state space into simplices, called a triangulation. Values are defined at each vertex of the triangulation and a continuous and piecewise affine function is then defined via a convex interpolation of these values. In [7], the vertex values are obtained by solving a linear programming problem that incorporates error estimates.

A similar CPA method has been developed for continuoustime systems [15] (see also [6]) where the need to solve

$\dagger$ S. Hafstein is with the School of Science and Engineering at Reykjavik University, Iceland sigurdurheru.is. $¥$ C.M. Kellett is with the School of Electrical Engineering and Computer Science, University of Newcastle, Callaghan, New South Wales, Australia Chris.Kellettanewcastle.edu.au. Kellett is supported by ARC Future Fellowship FT1101000746 and by the Alexander von Humboldt Foundation. * H. Li is with the Mathematical Institute, University of Bayreuth, 95440 Bayreuth, Germany Huijuan.Li@uni-bayreuth.de. Li is supported by the EU Initial Training Network "Sensitivity Analysis for Deterministic Controller Design-SADCO”. a linear programming problem results in long computation times. As an alternative to solving a linear programming problem, in [9] we proposed a new approach to compute a CPA Lyapunov function for continuous-time systems using a function in a converse Lyapunov theorem originally introduced by Yoshizawa in [18]. In this paper, we present a similar approach for the discrete-time nonlinear system described by

$$
x^{+}=g(x)
$$

where $g: \mathbb{R}^{n} \rightarrow \mathbb{R}^{n}$ is locally Lipschitz continuous, and $g(0)=0$. We observe that deriving such discrete-time results from their continuous-time counterparts is nontrivial due to the fact that solutions in the discrete-time setting are sequences of points rather than absolutely continuous functions as in the continuous-time setting.

The paper is organized as follows: we present the theory required for CPA Lyapunov functions in Section II. In Section III we formulate the required stability estimate and define the discrete-time Yoshizawa function. We also present the procedure to compute a CPA Lyapunov function for system (1) using the Yoshizawa function. In Section IV, we present three representative examples to demonstrate the proposed method. In Section V, we provide a brief summary.

\section{Continuous And Piecewise AfFine Lyapunov FUNCTIONS}

We denote the nonnegative integers by $\mathbb{N}^{0}$. Let $\mathbb{R}_{>0}$, $\mathbb{R}_{>0}$ denote intervals $(0,+\infty),[0,+\infty)$ respectively. Given a subset $\Omega \subset \mathbb{R}^{n}$, we denote the interior, the closure, the boundary, and the complement of $\Omega$ by $\Omega^{\circ}, \bar{\Omega}, \partial \Omega$, and $\Omega^{C}$, respectively. For $\delta \in \mathbb{R}_{>0}$, let $\mathcal{B}_{\delta} \doteq\left\{x \in \mathbb{R}^{n}:|x|<\delta\right\}$ denote the open ball of radius $\delta>0$ centered at 0 . Let $\lceil a\rceil \doteq \min \left\{q \in \mathbb{N}^{0}: q \geq a, a \in \mathbb{R}_{\geq 0}\right\}$ denote the smallest integer which is not less than $a$. The $k$ th element of the solution sequence of (1) with initial condition $x \in \Omega$ is denoted by $\phi(k, x)$ for all $k \in \mathbb{N}^{0}$ with $\phi(0, x)=x$.

In order to define CPA functions, we recall the definition of a suitable triangulation of a compact set from [9].

Definition 1: We call a finite collection $\mathcal{T}=\left\{\mathfrak{S}_{1}, \mathfrak{S}_{2}, \ldots, \mathfrak{S}_{N}\right\}$ of $n$-simplices in $\mathbb{R}^{n}$ a suitable triangulation if

i) $\mathfrak{S}_{\nu}, \mathfrak{S}_{\mu} \in \mathcal{T}, \nu \neq \mu$, intersect in a common face or not at all.

ii) With $\mathcal{D}_{\mathcal{T}} \doteq \cup_{\nu} \mathfrak{S}_{\nu}$, $\mathcal{D}_{\mathcal{T}}^{\circ}$ is a connected neighborhood of the origin.

iii) If $0 \in \mathfrak{S}_{\nu}$, then 0 is a vertex of $\mathfrak{S}_{\nu}$. 
In this paper, simplices are defined as the closed convex hull of an ordered set of vertices; i.e., for each $\nu \in\{1, \ldots, N\}$, for the ordered set of vectors $x_{i}^{\nu} \in \mathbb{R}^{n}, i=0, \ldots, n$, $x_{0}^{\nu}, \ldots, x_{n}^{\nu}$ are linearly independent, a simplex $\mathfrak{S}_{\nu}$ is defined by $\mathfrak{S}_{\nu}=\operatorname{co}\left\{x_{0}^{\nu}, \ldots, x_{n}^{\nu}\right\}$. Defining simplices in this way ensures that the shape matrix defined below in (14) is unique.

For a suitable triangulation $\mathcal{T}$, we define $\operatorname{CPA}[\mathcal{T}]$ as the set of continuous functions $f: \mathcal{D}_{\mathcal{T}} \rightarrow \mathbb{R}$ which are linearly affine on each simplex $\mathfrak{S}_{\nu}$; i.e.,

$$
f(x)=w_{\nu}^{\top} x+a_{\nu}, \quad x \in \mathfrak{S}_{\nu},
$$

where $w_{\nu} \in \mathbb{R}^{n}$ and $a_{\nu} \in \mathbb{R}$.

In the interior of any simplex, a function $f \in \mathrm{CPA}[T]$ is differentiable and we denote the gradient of a function $f \in \mathrm{CPA}[\mathcal{T}]$ in the interior of simplex $\mathfrak{S}_{\nu}$ by $\nabla f_{\nu}$. In other words, with (2), for each $x \in \mathfrak{S}_{\nu}^{\circ}$ we have

$$
\nabla f_{\nu} \doteq \nabla f(x)=w_{\nu}
$$

In the following, we present the definition of a CPA Lyapunov function for system (1) on a closed, connected set $\mathcal{O} \subset \mathcal{D}_{\mathcal{T}} \subset \mathbb{R}^{n}$ minus a fixed arbitrary small neighborhood of the origin.

Definition 2: Let $\mathcal{T}$ be a suitable triangulation, $V \in$ $\mathrm{CPA}[\mathcal{T}]$ be a positive definite function, and $\mathcal{O} \subset \mathcal{D}_{\mathcal{T}}$ be a closed, connected set such that

(i) $0 \in \mathcal{O} \subset \mathcal{D}_{\mathcal{T}}$;

(ii) there exists no $\mathfrak{S}_{\nu}$ with $x^{1}, x^{2} \in \mathfrak{S}_{\nu}$ satisfying $x^{1} \in$ $\mathcal{O}, x^{2} \in \mathcal{D}_{\mathcal{T}} \backslash \mathcal{O}$; and

(iii) if $x \in \mathcal{O}$, then $g(x) \in \mathcal{D}_{\mathcal{T}}$.

Define the constants

$$
\begin{aligned}
& \alpha_{1}^{*} \doteq \inf _{x \in \mathcal{O} \backslash\{0\}} \frac{V(x)}{|x|}, \alpha_{2}^{*} \doteq \sup _{x \in \mathcal{O} \backslash\{0\}} \frac{V(x)}{|x|}, \\
& q^{*}=\inf \left\{q \in \mathbb{R}_{\geq 0}:|g(x)| \leq q|x|, x \in \mathcal{O}\right\},
\end{aligned}
$$

and $r \doteq \min _{x \in \partial \mathcal{O}}|x|$. Let $\varepsilon \in \mathbb{R}_{>0}$ satisfy

$$
\left\{\begin{array}{c}
0<q^{*} \varepsilon<r \alpha_{1}^{*} / \alpha_{2}^{*} \\
\mathcal{B}_{q^{*} \varepsilon} \subset \mathcal{O}
\end{array} \quad, \quad \text { for } q^{*} \geq 1\right.
$$

or

$$
0<\varepsilon<r \alpha_{1}^{*} / \alpha_{2}^{*}, \text { for } q^{*}<1
$$

If there is a constant $\alpha_{3}^{*} \in \mathbb{R}_{>0}$ such that

$$
V(g(x))-V(x) \leq-\alpha_{3}^{*}|x|
$$

holds for all $x \in \mathcal{O} \backslash \mathcal{B}_{\varepsilon}$, then $V$ is called a $\mathrm{CPA}[\mathcal{T}]$ Lyapunov function for (1) on $\mathcal{O} \backslash \mathcal{B}_{\varepsilon}$.

By a slight abuse of notation we denote the set of solutions of (1) at time $k \in \mathbb{N}^{0}$ from a compact set $\mathcal{C} \subset \mathbb{R}^{n}$ by $\phi(k, \mathcal{C}):=\bigcup_{x \in \mathcal{C}} \phi(k, x)$. Denote the sublevel sets of $V$ by

$$
\mathcal{L}_{V, c} \doteq\left\{x \in \mathcal{D}_{\mathcal{T}}: V(x) \leq c\right\}, \quad c \in \mathbb{R}_{>0} .
$$

Theorem 1: Let $\mathcal{T}$ be a suitable triangulation and let $V: \mathcal{D}_{\mathcal{T}} \rightarrow \mathbb{R}_{\geq 0}$ be a $\mathrm{CPA}[\mathcal{T}]$ Lyapunov function for $(1)$ on $\mathcal{O} \backslash \mathcal{B}_{\varepsilon}$ with appropriate $\alpha_{1}^{*}, \alpha_{2}^{*}, q^{*}, r, \varepsilon \in \mathbb{R}_{>0}$ as in
Definition 2 and where $\mathcal{O} \subset \mathbb{R}^{n}$ satisfies conditions (i)-(iii) from Definition 2. Define

$$
m \doteq\left\{\begin{array}{c}
\max _{|x| \leq q^{*} \varepsilon} V(x), \text { if } \quad q^{*} \geq 1 \\
\max _{|x| \leq \varepsilon} V(x), \text { if } \quad q^{*}<1
\end{array}\right.
$$

and $M \doteq \min _{x \in \partial \mathcal{O}} V(x)$. If for each $c \in[m, M), \mathcal{L}_{V, c}$ is connected, then $\mathcal{B}_{\varepsilon} \subset \mathcal{L}_{V, c} \subset \mathcal{O}$ and there exists a $K_{c} \in \mathbb{N}^{0}$ such that $\phi\left(k, \mathcal{L}_{V, c}\right) \subset \mathcal{L}_{V, m}$ for all $k \geq K_{c}$.

Proof: We first prove $\mathcal{B}_{\varepsilon} \subset \mathcal{L}_{V, m}$. Given $x \in \mathbb{R}^{n}$ satisfying $|x| \leq \varepsilon, x \neq 0$, and $y \in \partial \mathcal{O}$, then

$$
V(x)=|x| \frac{V(x)}{|x|} \leq|x| \sup _{x \in \mathcal{O} \backslash\{0\}} \frac{V(x)}{|x|}=|x| \alpha_{2}^{*} \leq \varepsilon \alpha_{2}^{*},
$$

and by (6) and (7),

$$
V(x)<\alpha_{1}^{*} r \leq V(y)
$$

If $q^{*} \geq 1$, the constraint (6) implies (12) holds for $x$ satisfying $|x| \leq q^{*} \varepsilon$. Based on the definition of $m$, then we obtain $m<M$.

It follows directly by the definitions of $m$ and $M$ and the continuity of $V$ that if $\mathcal{L}_{V, c}$ is connected then $\mathcal{B}_{\varepsilon} \subset \mathcal{L}_{V, c} \subset$ $\mathcal{O}$. For $x \in \mathcal{L}_{V, c} \backslash \mathcal{B}_{\varepsilon}$ we get by (8) that $g(x) \in \mathcal{L}_{V, c}$. For $x \in \mathcal{B}_{\varepsilon}$ we get by (5) that $|g(x)|<q^{*} \varepsilon$. Hence, by the definition of $m$ we get $g(x) \in \mathcal{L}_{V, m} \subset \mathcal{L}_{V, c}$. Thus $\mathcal{L}_{V, c}$ is positively invariant. The last assertion of the theorem now follows from (8) with $K_{c} \geq(c-m) /\left(\alpha_{3} \varepsilon\right)$.

Remark 1: The conditions of Theorem 1 are more restrictive than those in [9, Theorem 2.3]. These more restrictive conditions are required because the solution of (1) is a sequence of points rather than an absolutely continuous function. Similar to the continuous time result in [9, Theorem 2.3] Theorem 1 provides an estimate of the domain of attraction for the positively invariant set $\mathcal{L}_{V, m}$.

We state the criteria for verifying that a CPA function is a CPA Lyapunov function in Theorem 2 and Corollary 1.

Theorem 2: Let $\mathcal{T}$ be a suitable triangulation and let $a$ closed, connected set $\mathcal{O} \subset \mathbb{R}^{n}$ satisfy conditions (i)-(iii) from Definition 2. Let $V \in \mathrm{CPA}[\mathcal{T}], \varepsilon \in \mathbb{R}_{>0}$, and let $C, L_{\nu} \in$ $\mathbb{R}_{\geq 0}$ satisfy

$$
\begin{aligned}
& |g(x)-g(y)| \leq L_{\nu}|x-y|, \text { for } x, y \in \mathfrak{S}_{\nu}, \\
& \max _{\nu=1, \ldots, N}\left|\nabla V_{\nu}\right| \leq C .
\end{aligned}
$$

If for each $\mathfrak{S}_{\nu} \subset \mathcal{O}, \mathfrak{S}_{\nu} \cap \mathcal{B}_{\varepsilon}^{C}=\emptyset$ the inequalities

$$
V\left(g\left(x_{i}^{\nu}\right)\right)-V\left(x_{i}^{\nu}\right)+C L_{\nu} \operatorname{diam}\left(\mathfrak{S}_{\nu}\right)<0
$$

hold for all $i=0,1, \ldots, n$, then

$$
V(g(x))-V(x)<0
$$

for all $x \in \mathcal{O} \backslash \mathcal{B}_{\varepsilon}$.

Proof: Let $x \in \mathcal{O} \backslash \mathcal{B}_{\varepsilon}$ be arbitrary. Then there exists a $\mathfrak{S}_{\nu} \subset \mathcal{O}$ such that $x \in \mathfrak{S}_{\nu}$; i.e., $x=\sum_{i=0}^{n} \lambda_{i} x_{i}^{\nu}$ where 
$\sum_{i=0}^{n} \lambda_{i}=1$. Then

$$
\begin{array}{r}
V(g(x))-V(x)=V(g(x))-\sum_{i=0}^{n} \lambda_{i} V\left(g\left(x_{i}^{\nu}\right)\right) \\
+\sum_{i=0}^{n} \lambda_{i} V\left(g\left(x_{i}^{\nu}\right)\right)-\sum_{i=0}^{n} \lambda_{i} V\left(x_{i}^{\nu}\right) \\
\leq \sum_{i=0}^{n} \lambda_{i}\left[V\left(g\left(x_{i}^{\nu}\right)\right)-V\left(x_{i}^{\nu}\right)+C L_{\nu} \operatorname{diam}\left(\mathfrak{S}_{\nu}\right)\right] .
\end{array}
$$

Based on (13), we conclude that $V(g(x))-V(x)<0$ for all $x \in \mathcal{O} \backslash \mathcal{B}_{\varepsilon}$.

Corollary 1: Let $V \in \mathrm{CPA}[\mathcal{T}]$ from Theorem 2 be positive definite and the constant $\varepsilon \in \mathbb{R}_{>0}$ satisfy (6) or (7) as appropriate. If the inequalities (13) are satisfied for all $\mathfrak{S}_{\nu} \subset \mathcal{O}$ with $\mathfrak{S}_{\nu} \cap \mathcal{B}_{\varepsilon}^{C} \neq \emptyset$, then $V$ is a CPA Lyapunov function for (1) on $\mathcal{O} \backslash \mathcal{B}_{\varepsilon}$.

Remark 2: From Theorem 2 and Corollary 1, for a candidate Lyapunov function $V \in \mathrm{CPA}[\mathcal{T}]$, the verification that $V$ is a Lyapunov function for system (1) is done by checking that $V$ is positive definite and that the inequality (13) holds for each vertex. The problem then is to find a candidate Lyapunov function. In order to obtain a CPA candidate Lyapunov function, [7] obtains a CPA function by solving a linear programming problem. In the present paper, we compute the value at each vertex by using a particular function from a converse Lyapunov theorem, called a Yoshizawa function (see (28) below), and then verify the inequality (13) for each vertex.

In the following we recall the definition of CPA approximations to functions as stated in [9, Definition 2.6].

Definition 3: Let $\mathcal{D} \subset \mathbb{R}^{n}$ be a domain, $g: \mathcal{D} \rightarrow \mathbb{R}$ be a function, and $\mathcal{T}$ be a triangulation such that $\mathcal{D}_{\mathcal{T}} \subset \mathcal{D}$. The CPA $[\mathcal{T}]$ approximation $f$ to $g$ on $\mathcal{D}_{\mathcal{T}}$ is the function $f \in \mathrm{CPA}[\mathcal{T}]$ defined by $f(x)=g(x)$ for all vertices $x$ of all simplices in $\mathcal{T}$.

Given a triangulation $\mathcal{T}$, and simplex $\mathfrak{S}_{\nu} \doteq$ $\operatorname{co}\left\{x_{0}^{\nu}, x_{1}^{\nu}, \ldots, x_{n}^{\nu}\right\} \in \mathcal{T}$, the shape-matrix $X_{\nu}$ of $\mathfrak{S}_{\nu}$ is defined by writing the vectors $x_{1}^{\nu}-x_{0}^{\nu}, x_{2}^{\nu}-x_{0}^{\nu}, \ldots, x_{n}^{\nu}-x_{0}^{\nu}$ in its rows subsequently; i.e.,

$$
X_{\nu}=\left[\left(x_{1}^{\nu}-x_{0}^{\nu}\right),\left(x_{2}^{\nu}-x_{0}^{\nu}\right), \cdots,\left(x_{n}^{\nu}-x_{0}^{\nu}\right)\right]^{\top} .
$$

In order to make sure that the simplex $\mathfrak{S}_{\nu}$ is not degenerate, the value $\operatorname{diam}\left(\mathfrak{S}_{\nu}\right)\left|X_{\nu}^{-1}\right|$ should be bounded. Here $\left|X_{\nu}^{-1}\right|$ is the spectral norm of the inverse of $X_{\nu}$ (see part (ii) in the proof of [2, Theorem 4.6]).

Definition 4: Given a domain $\mathcal{D} \subset \mathbb{R}^{n}$, a continuous function $W: \mathbb{R}^{n} \rightarrow \mathbb{R}_{\geq 0}$ is a Lyapunov function for (1) on $\mathcal{D}$ if there exist positive definite functions $\alpha, \alpha_{1}: \mathbb{R}_{\geq 0} \rightarrow \mathbb{R}_{\geq 0}$ such that, for every $x \in \mathcal{D}$,

$$
\begin{aligned}
\alpha_{1}(|x|) & \leq W(x), \text { and } \\
W(g(x)) & -W(x) \leq-\alpha(|x|) .
\end{aligned}
$$

We now state conditions under which the CPA approximation to a Lyapunov function is also a Lyapunov function.

Theorem 3: Let $\mathcal{O}, \mathcal{D} \subset \mathbb{R}^{n}$ be simply connected compact neighborhoods of the origin such that $\overline{\mathcal{O}^{\circ}}=\mathcal{O}, \overline{\mathcal{D}^{\circ}}=\mathcal{D}$,
$\mathcal{O} \subset \mathcal{D}^{\circ}$. Further, assume that $W \in C^{1}(\mathcal{D})$ is a Lyapunov function for system (1) and there exists a constant $L>0$ such that $|\nabla W(x)| \leq L$ for $x \in \mathcal{D}$. Set $\tilde{r} \doteq \min _{x \in \partial \mathcal{O}}|x|$ and let $\varepsilon \in \mathbb{R}_{>0}$ satisfy

$$
\begin{gathered}
q^{*} \varepsilon<\tilde{r} \cdot \frac{\inf _{x \in \mathcal{O} \backslash\{0\}} W(x) /|x|}{\sup _{x \in \mathcal{O} \backslash\{0\}} W(x) /|x|} \\
\mathcal{B}_{q^{*} \varepsilon} \subset \mathcal{O}
\end{gathered} \quad, \text { for } q^{*} \geq 1,
$$

where $q^{*}$ from Definition 2.

Then for every $R>0$ there exists a $\delta_{R}>0$ such that, for any triangulation $\mathcal{T}$ satisfying

1) $\mathcal{O} \subset \mathcal{D}_{\mathcal{T}} \subset \mathcal{D}$,

2) $x \in \mathcal{O}$ implies $g(x) \in \mathcal{D}_{\mathcal{T}}$,

3) there exists no $\mathfrak{S}_{\nu}$ with $x^{1}, x^{2} \in \mathfrak{S}_{\nu}$ satisfying $x^{1} \in \mathcal{O}$, $x^{2} \in \mathcal{D}_{\mathcal{T}} \backslash \mathcal{O}$,

4) $\max _{\mathfrak{S}_{\nu} \in \mathcal{T}} \operatorname{diam}\left(\mathfrak{S}_{\nu}\right) \leq \delta_{R}$, and

5) $\max _{\mathfrak{S}_{\nu} \in \mathcal{T}} \operatorname{diam}\left(\mathfrak{S}_{\nu}\right)\left|X_{\nu}^{-1}\right| \leq R$,

the $\mathrm{CPA}[\mathcal{T}]$ approximation $V$ to $W$ on $\mathcal{D}_{\mathcal{T}}$ is a CPA Lyapunov function for (1) on $\mathcal{O} \backslash \mathcal{B}_{\mathcal{\varepsilon}}$.

Proof: Since $W(x)$ is a Lyapunov function for (1) on $\mathcal{D}$, we get that there exists a positive definite function $\alpha$ : $\mathbb{R}_{\geq 0} \rightarrow \mathbb{R}_{\geq 0}$ such that

$$
W(g(x))-W(x) \leq-\alpha(|x|), \text { for } x \in \mathcal{D} .
$$

Let

$$
\chi=\max _{\nu=1,2, \ldots, N}\left|X_{\nu}^{-1}\right|=\max _{\nu=1,2, \ldots, N} \lambda_{\min , \nu}^{-\frac{1}{2}}
$$

where $\lambda_{\min , \nu}$ is the minimum eigenvalue of $X_{\nu}^{\top} X_{\nu}$.

For an arbitrary but fixed $\mathfrak{S}_{\nu}=\operatorname{co}\left\{x_{0}^{\nu}, x_{1}^{\nu}, \cdots, x_{n}^{\nu}\right\} \subset$ $\mathcal{D}_{\mathcal{T}} \backslash \mathcal{B}_{\varepsilon}$ define

$$
W_{\nu}:=\left(\begin{array}{c}
W\left(x_{1}^{\nu}\right)-W\left(x_{0}^{\nu}\right) \\
W\left(x_{2}^{\nu}\right)-W\left(x_{0}^{\nu}\right) \\
\vdots \\
W\left(x_{n}^{\nu}\right)-W\left(x_{0}^{\nu}\right)
\end{array}\right) .
$$

For each vertex $x_{i}^{\nu} \in \mathfrak{S}_{\nu} \subset \mathcal{D}_{\mathcal{T}}$, let $V\left(x_{i}^{\nu}\right)=W\left(x_{i}^{\nu}\right)$. It is obvious that $V\left(x_{i}^{\nu}\right)$ is positive definite for $x_{i}^{\nu} \in \mathfrak{S}_{\nu} \subset \mathcal{D}_{\mathcal{T}}$.

Choose one $\mathfrak{S}_{\nu}=\operatorname{co}\left\{x_{0}^{\nu}, x_{1}^{\nu}, \cdots, x_{n}^{\nu}\right\} \subset \mathcal{D}_{\mathcal{T}} \backslash \mathcal{B}_{\varepsilon}$ and let $y=x_{0}^{\nu}$ and $x \in \mathfrak{S}_{\nu}$. Since $V \in \operatorname{CPA}[\mathcal{T}], V(x)=$ $V(y)+\nabla V_{\nu}^{\top}(x-y)$. Then taking $x=x_{i}^{\nu} \in \mathfrak{S}_{\nu}$ for all $i \in\{1, \cdots, n\}$, using the fact that $V\left(x_{i}^{\nu}\right)=W\left(x_{i}^{\nu}\right)$, and the definitions $W_{\nu},(19)$, and $X_{\nu},(14)$, we get

$$
\nabla V_{\nu}=X_{\nu}^{-1} W_{\nu} .
$$

Hence

$$
V(x)=V(y)+W_{\nu}^{\top}\left(X_{\nu}^{\top}\right)^{-1}(x-y) .
$$

There exists a constant $C=R L \in \mathbb{R}_{>0}$ such that

$$
\begin{aligned}
\left|\nabla V_{\nu}\right| & =\left|X_{\nu}^{-1} W_{\nu}\right| \leq\left|X_{\nu}^{-1}\right| \max _{z \in \mathcal{D}_{\mathcal{T}} \backslash \mathcal{B}_{\varepsilon}}|\nabla W(z)| \delta_{R} \\
& \leq R \max _{z \in \mathcal{D}_{\mathcal{T}} \backslash \mathcal{B}_{\varepsilon}}|\nabla W(z)| \leq C
\end{aligned}
$$

holds uniformly in $\nu$. 
Let $x_{i}^{\nu}$ be an arbitrary vertex of an arbitrary simplex $\mathfrak{S}_{\nu} \subset \mathcal{O}$. Since $g(x) \in \mathcal{D}_{\mathcal{T}}$, there exists an $\mathfrak{S}_{\mu} \doteq$ $\operatorname{co}\left\{y_{0}^{\mu}, y_{1}^{\mu}, \ldots, y_{n}^{\mu}\right\} \in \mathcal{T}$ such that $g\left(x_{i}^{\nu}\right)=\sum_{j=0}^{n} \mu_{j} y_{j}^{\mu} \in \mathfrak{S}_{\mu}$ with $\sum_{j=0}^{n} \mu_{j}=1$. We have assigned $V(x)=W(x)$ for all vertices $x$ of all simplices $\mathfrak{S}_{\nu}$. Hence

$$
\begin{aligned}
V\left(g\left(x_{i}^{\nu}\right)\right)-V\left(x_{i}^{\nu}\right) & =\sum_{j=0}^{n} \mu_{j} W\left(y_{j}^{\mu}\right)-W\left(x_{i}^{\nu}\right) \\
& =\sum_{j=0}^{n} \mu_{j} W\left(y_{j}^{\mu}\right)-W\left(\sum_{j=0}^{n} \mu_{j} y_{j}^{\mu}\right) \\
& +W\left(\sum_{j=0}^{n} \mu_{j} y_{j}^{\mu}\right)-W\left(x_{i}^{\nu}\right) .
\end{aligned}
$$

It follows that

$$
V\left(g\left(x_{i}^{\nu}\right)\right)-V\left(x_{i}^{\nu}\right) \leq L \delta_{R}-\alpha\left(\left|x_{i}^{\nu}\right|\right),
$$

It is obvious that for every $R>0$ there exists a suitable $\delta_{R}>0$ such that

$$
L \delta_{R}-\alpha\left(\left|x_{i}^{\nu}\right|\right)+C L_{\nu} \delta_{R}<0
$$

holds for all $x_{i}^{\nu} \in \mathcal{O} \backslash \mathcal{B}_{\varepsilon}$. Therefore the linear constraints (13) are fulfilled for all $x_{i}^{\nu} \in \mathcal{O} \backslash \mathcal{B}_{\varepsilon}$.

Based on the conditions, we obtain

$$
\begin{aligned}
& \inf _{x \in \mathcal{O} \backslash\{0\}} \frac{W(x)}{|x|} \leq \inf _{x^{\nu} \in \mathcal{O} \backslash\{0\}} \frac{V\left(x^{\nu}\right)}{\left|x^{\nu}\right|}=\inf _{x \in \mathcal{O} \backslash\{0\}} \frac{V(x)}{|x|}=\alpha_{1}^{*}, \\
& \sup _{x \in \mathcal{O} \backslash\{0\}} \frac{W(x)}{|x|} \geq \sup _{x^{\nu} \in \mathcal{O} \backslash\{0\}} \frac{V\left(x^{\nu}\right)}{\left|x^{\nu}\right|}=\sup _{x \in \mathcal{O} \backslash\{0\}} \frac{V(x)}{|x|}=\alpha_{2}^{*},
\end{aligned}
$$

and $r=\tilde{r}$, where $\alpha_{1}^{*}, \alpha_{2}^{*}, r \in \mathbb{R}_{>0}$ from Definition 2 . Therefore $\varepsilon$ satisfies conditions in Definition 2 and then the theorem is proved by Corollary 1 .

Remark 3: Since a given triangulation $\mathcal{T}$ can be manipulated to deliver a new triangulation $\mathcal{T}^{*}$ with smaller simplices without increasing their degeneracy, it is always possible to find a triangulation that admits a CPA Lyapunov function approximating a differentiable and Lipschitz continuous Lyapunov function.

\section{Yoshizawa CONSTRUCTION OF LyaPUnOV FUNCTIONS}

We now address the problem of how to calculate the vertex values for each simplex. Based on converse Lyapunov function theorems such as [1, Theorem 5.12.5], [8], [13], and [17, Theorem 1.7.6], if system (1) is asymptotically stable, then there exists a Lyapunov function. Among these results, we are interested in a particular construction of the Lyapunov function which was originally proposed in [18] in continuous-time and extended to the discrete-time case in [13]. In what follows, we will make use of the common function classes $\mathcal{K}_{\infty}$ and $\mathcal{K} \mathcal{L}$. For details of these functions, we refer to [10], [12].
Given an open compact set $\mathcal{D}$ with $0 \in \mathcal{D}$. System (1) is said to be $\mathcal{K} \mathcal{L}$-stable on $\mathcal{D}$ if there exists a $\beta \in \mathcal{K} \mathcal{L}$ such that

$$
|\phi(k, x)| \leq \beta(|x|, k), \quad \forall x \in \mathcal{D}, k \in \mathbb{N}^{0} .
$$

It has been proved in [14, Proposition 2.2] that the concept of $\mathcal{K} \mathcal{L}$-stability is equivalent to the the concept of asymptotic stability of the origin for system (1), given $\mathcal{D}$ is a subset of the domain of attraction. If $\mathcal{D}=\mathbb{R}^{n}$, then $\mathcal{K} \mathcal{L}$-stability is equivalent to global asymptotic stability of the origin for system (1). The function $\beta \in \mathcal{K} \mathcal{L}$ of (26) is called a stability estimate.

In order to define our candidate Lyapunov function, we use a version of Sontag's lemma on $\mathcal{K} \mathcal{L}$-estimates [16, Proposition 7].

Lemma 1: For every $\mu \in(0,1), \beta \in \mathcal{K} \mathcal{L}$ there exist $\alpha_{1}, \alpha_{2} \in \mathcal{K}_{\infty}$ such that

$$
\alpha_{1}(\beta(s, k)) \leq \alpha_{2}(s) \mu^{2 k}, \forall s \in \mathbb{R}_{\geq 0}, \forall k \in \mathbb{N}^{0} .
$$

Proof: Sontag's lemma on $\mathcal{K} \mathcal{L}$-estimates [16, Proposition 7] states that, for any $\lambda>0$ and $\beta \in \mathcal{K} \mathcal{L}$ there exist $\alpha_{1}, \alpha_{2} \in \mathcal{K}_{\infty}$ so that

$$
\alpha_{1}(\beta(s, k)) \leq \alpha_{2}(s) \mu^{2 k}, \forall s \in \mathbb{R}_{\geq 0}, \forall k \in \mathbb{N}^{0} .
$$

Given $\mu \in(0,1)$, let $\lambda=-2 \log \mu$ which satisfies $\lambda>0$. Applying [16, Proposition 7] with this $\lambda>0$ then yields (27).

We now define the discrete-time Yoshizawa function.

Definition 5: Given $\mu \in(0,1)$ and $\beta \in \mathcal{K} \mathcal{L}$ with $\alpha_{1}, \alpha_{2} \in$ $\mathcal{K}_{\infty}$ from Lemma 1 , the function

$$
V(x) \doteq \sup _{k \in \mathbb{N}^{0}} \alpha_{1}(|\phi(k, x)|) \mu^{-k}
$$

is called a discrete-time Yoshizawa function.

Based on the results of [14], we summarize some properties of the discrete-time Yoshizawa function in the next theorem.

Theorem 4: If the solution $\phi(k, x)$ of system (1) with $x \in$ $\mathcal{D}$ satisfies (26), and $\alpha_{1}(\cdot)$ is locally Lipschitz continuous, then the Yoshizawa function is continuous on $\mathcal{D} \backslash\{0\}$ and satisfies the bounds

$$
\alpha_{1}(|x|) \leq V(x) \leq \alpha_{2}(|x|)
$$

and the decrease condition

$$
V(\phi(1, x)) \leq V(x) \mu .
$$

Further, for each $x \in \mathcal{D}$ there exists a positive integer $K(x)$ such that

$$
V(x)=\max _{k \in\{0, \cdots, K(x)\}} \alpha_{1}(|\phi(k, x)|) \mu^{-k} .
$$

The properties that $V(x)$ is continuous, bounded and satisfies the decrease condition have been proved in [14].

Let $\lambda=\mu^{-1}$, the integer $K(x)$ is calculated explicitly in [14, Claim 7] as

$$
K(x)=\left\lceil-\log _{\lambda}\left(\frac{V(x)}{\alpha_{2}(|x|)}\right)\right\rceil+1, \quad x \neq 0
$$


and with the upper and lower bounds on $V$ we get that

$$
\begin{aligned}
0 \leq K(x) & \leq\left\lceil-\log _{\lambda}\left(\frac{\alpha_{1}(|x|)}{\alpha_{2}(|x|)}\right)\right]+1 \\
& =\left\lceil\log _{\lambda}\left(\frac{\alpha_{2}(|x|)}{\alpha_{1}(|x|)}\right)\right]+1 \doteq \overline{K(x)} .
\end{aligned}
$$

In the computations in the following section, we use $\overline{K(x)}$ instead of $K(x)$ in the computation of (31). This is done since we do not need to know $V(x)$ to calculate $\overline{K(x)}$ and, from the relations (28) and (31), taking a longer time horizon in (31) will not change the value of $V(x)$. Examples of the use of Sontag's lemma on $\mathcal{K} \mathcal{L}$-estimates to define the Yoshizawa function can be found in [9, Example 1, Example 2].

For system (1), our proposed approach of constructing a CPA Lyapunov function is the following:

1: Obtain a stability estimate $\beta \in \mathcal{K} \mathcal{L}$ so that (26) holds.

2: Find $\alpha_{1}, \alpha_{2} \in \mathcal{K}_{\infty}$ satisfying inequality (27).

3: Define a suitable triangulation on a subset of the state space containing the equilibrium.

4: Calculate the vertex values of each simplex via Yoshizawa function defined by (28).

5: Construct a CPA function via convex interpolation of the vertex values of each simplex.

6: Check inequality (13) for each vertex.

Note that for computational reasons, we exclude a small neighborhood of the equilibrium when constructing the triangulation.

From Theorem 2 and Corollary 1, if (13) holds for each vertex, then such a CPA function is a Lyapunov function. If the Yoshizawa function (28) is a differentiable function and Lipschitz continuous, based on Theorem 3 our method always succeeds on each subset of the domain of attraction. However, from Theorem 4 the Yoshizawa function (28) is only continuous. Thus a subject of future work is to investigate under what conditions the Yoshizawa function (28) is differentiable.

\section{NumERICAL EXAMPLES}

In this section we present three examples to demonstrate the effectiveness of the proposed method.

\section{A. Example 1 - Linear System}

Consider the system

$$
x^{+}=A x=\left[\begin{array}{cc}
0.25 & 0.25 \\
-0.125 & -0.25
\end{array}\right] x
$$

Let $x=\left(x^{1}, x^{2}\right)^{\top}$. We observe that the origin is globally exponentially stable as the eigenvalues of $A$ are at $\pm \frac{\sqrt{2}}{8}$. We solve the so-called discrete Lyapunov equation,

$$
A^{\top} P A=P-0.25 \mathrm{Id}
$$

where $P$ is a symmetric positive definite matrix and thus obtain that

$$
V(x)=x^{\top} P x=x^{\top}\left[\begin{array}{cc}
0.2815 & -0.0235 \\
-0.0235 & -0.2698
\end{array}\right] x
$$

is a Lyapunov function as shown in Figure 2 for system (34).

We observe that

$$
|\phi(k, x)| \leq\left(\frac{\sqrt{2}}{8}\right)^{k}|x| \leq e^{-k}|x|
$$

and so (34) has a stability estimate $\beta \in \mathcal{K} \mathcal{L}$ given by

$$
\beta(s, k)=s e^{-k} .
$$

With $\alpha_{1}(s)=s^{2}=\alpha_{2}(s)$, then $\overline{K(x)}=1$. Therefore

$$
V_{1}(x)=\max _{k \in\{0,1\}} \alpha_{1}(|\phi(k, x)|) e^{k}
$$

Figure 1 shows the computed CPA Lyapunov function $V_{1}(x)$ for system (34). The neighborhood of the origin $\mathcal{B}_{\varepsilon}, \varepsilon=$ 0.05 , is excluded.

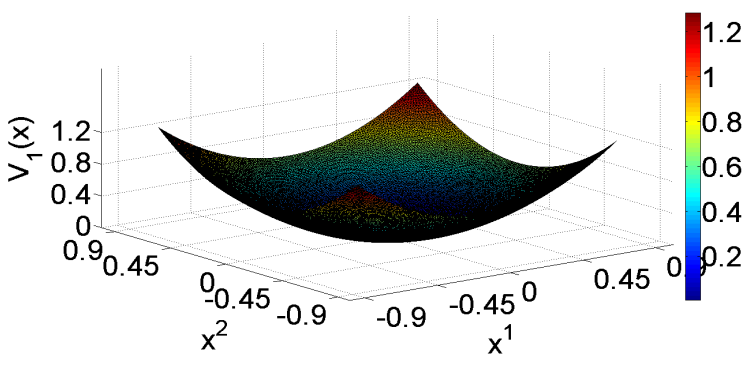

Fig. 1. CPA Lyapunov function $V_{1}(x)$ for system (34).

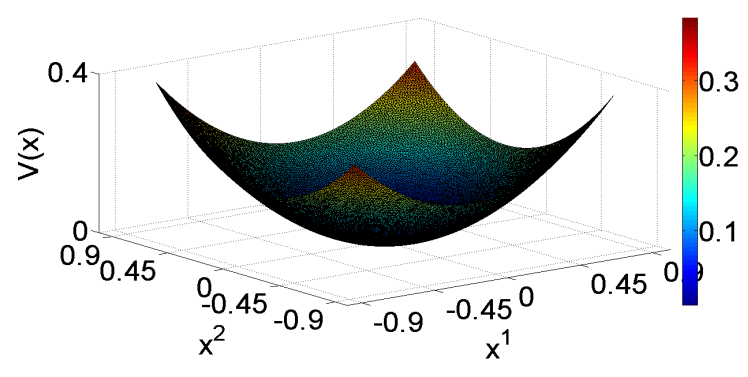

Fig. 2. Lyapunov function $V(z)$ for system (34).

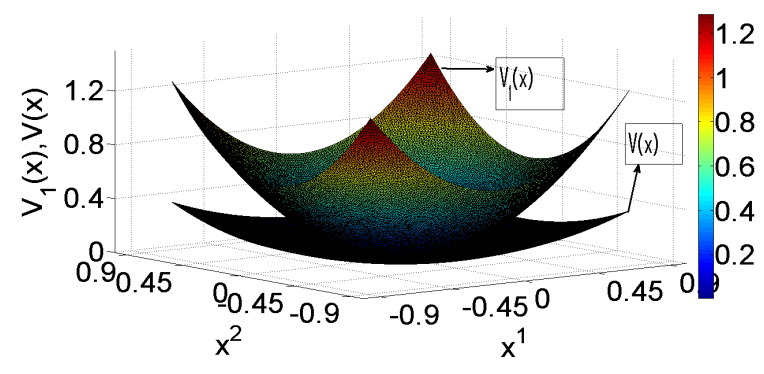

Fig. 3. Lyapunov function $V(x), V_{1}(x)$ for system (34). 


\section{B. Example 2 - Simple Nonlinear System}

Consider the one-dimensional system

$$
x^{+}=\left\{\begin{array}{cll}
\frac{1}{2} x^{2}, & \text { if } & |x| \leq 1 \\
\frac{1}{2} \sqrt{|x|}, & \text { if } & |x|>1
\end{array}\right.
$$

It is obvious that $\left|x^{+}\right| \leq \frac{1}{2}|x|$. Let $\mu=\frac{\sqrt{2}}{2}, \alpha_{1}(s)=\alpha_{2}(s)=$ $s$. Then $\overline{K(x)}=1$ and

$$
|\phi(k, x)| \leq\left(\frac{1}{2}\right)^{k}|x| \leq|x| \mu^{2 k} .
$$

It follows that

$$
V_{2}(x)=\max _{k \in\{0,1\}} \alpha_{1}(|\phi(k, x)|) \mu^{-k}
$$

which is shown by Figure 4 for system (39) for $5>|x| \geq$ 0.025 .

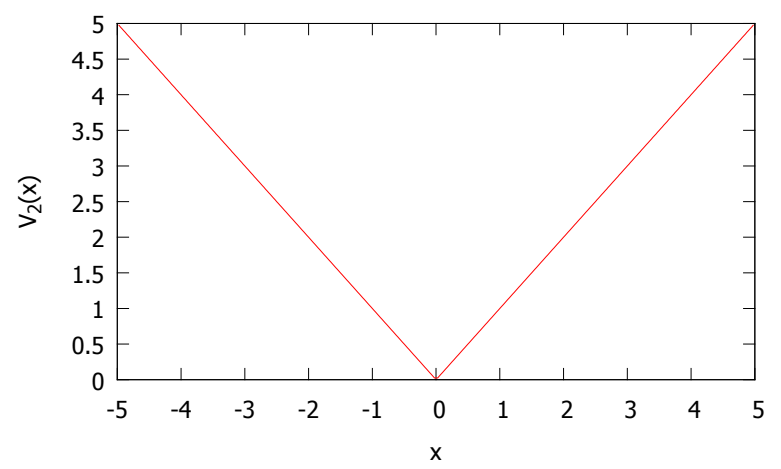

Fig. 4. Lyapunov function $V_{2}(x)$ for system (39).

\section{Example 3 - Nonlinear System}

Consider the two-dimensional nonlinear system described by

$$
\begin{aligned}
& x^{+}=-0.125 y-0.125\left(1-x^{2}-y^{2}\right) x, \\
& y^{+}=0.125 x-0.125\left(1-x^{2}-y^{2}\right) y .
\end{aligned}
$$

Let $z \doteq(x, y)^{\top}$. For $|z|<1$, it is easy to get that $\left|z^{+}\right| \leq$ $\frac{\sqrt{2}}{8}|z|$. Like Example 1, the stability estimate is given by

$$
|\phi(k, z)| \leq|z| e^{-k}
$$

Let $\alpha_{1}(s)=s^{2}=\alpha_{2}(s)$, then $\overline{K(z)}=1$. Thus

$$
V_{3}(z)=\max _{k \in\{0,1\}} \alpha_{1}(|\phi(k, z)|) e^{k} .
$$

Figure 5 shows the computed CPA Lyapunov function for system (42) for $0.8>|z| \geq 0.05$. On any compact subset of the unit ball, the simple quadratic

$$
V(z)=x^{2}+y^{2}
$$

is a known Lyapunov function which is shown by Figure (6).

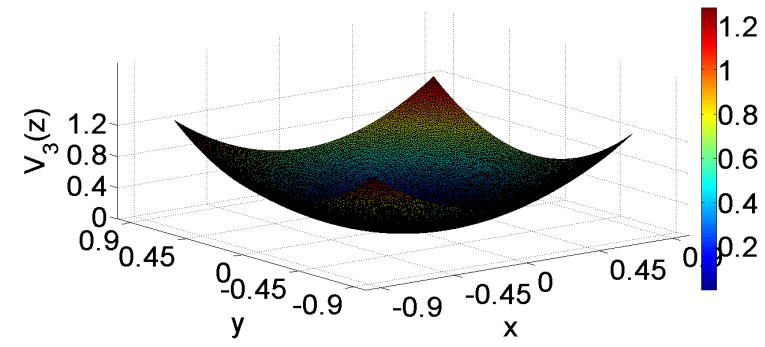

Fig. 5. Lyapunov function $V(z)$ for system (42).

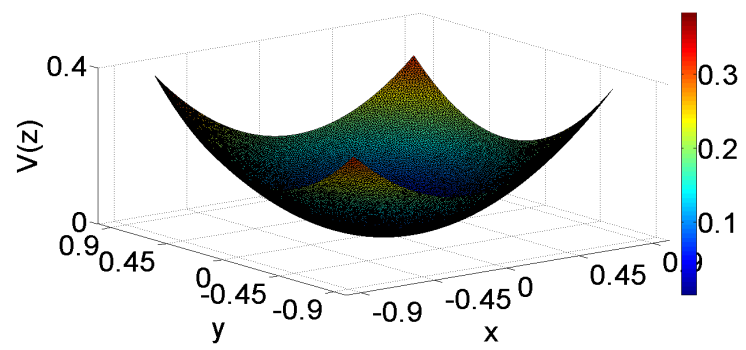

Fig. 6. Lyapunov function $V(z)$ for system (42).

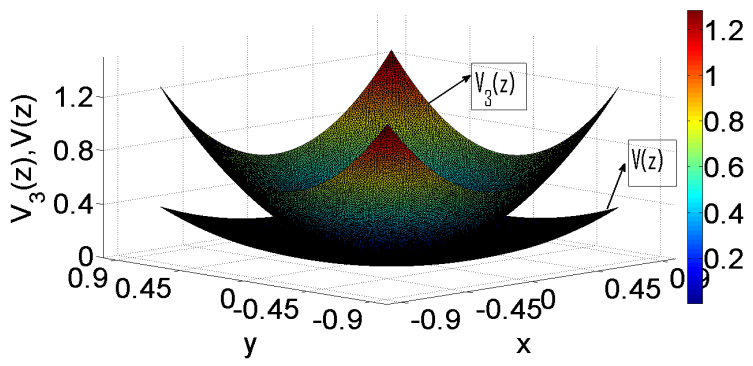

Fig. 7. Difference between Lyapunov functions $V_{3}(z)$ and $V(z)$ for system (42).

\section{Conclusions}

In this paper, we proposed a new method of computing a CPA Lyapunov function for discrete-time dynamic system (1). This approach replaces the linear program of [15] and [6] with evaluation of the Yoshizawa function at the vertices of the triangulation. We observe that in the numerical examples presented in Section IV computing the Yoshizawa function only requires taking the maximum between two easily computable values, making this a very efficient method to obtain vertex values. If (1) is $\mathcal{K} \mathcal{L}$ stable, our approach successfully delivers a CPA Lyapunov function. However, for general discrete-time dynamic systems, there is no explicit procedure to obtain a $\mathcal{K} \mathcal{L}$ stability estimate. Even if a $\mathcal{K} \mathcal{L}$ stability estimate is known, finding suitable $\alpha_{1}, \alpha_{2}$ satisfying (27) is not necessarily straightforward and the proof of [16, Proposition 7] is not constructive.

\section{REFERENCES}

[1] R. P. Agarwal. Difference Equations and Inequalities: Theory, Methods, and Applications. Marcel Dekker, 2 edition, 2000. 
[2] R. Baier, L. Grüne, and S. Hafstein. Linear programming based Lyapunov function computation for differential inclusions. Discrete and Continuous Dynamical Systems Series B, 17:33-56, 2012.

[3] H. Ban and W. D. Kalies. A computational approach to conley's decomposition theroem. J. Comput. Nonlinear Dynam., (1-4):312319, 2006

[4] P. Giesl. On the determination of the basin of attraction of discrete dynamical systems. Journal of Difference Equations and Applications, 13:523-546, 2007.

[5] P. Giesl. Construction of a local and global Lyapunov function using radial basis functions. IMA J. Appl. Math., 73(5):782-802, 2008.

[6] P. Giesl and S. Hafstein. Revised CPA method to compute Lyapunov functions for nonlinear systems. Journal of Mathematical Analysis and Applications, 410:292-306, 2014.

[7] P. Giesl and S. Hafstein. Computation of Lyapunov functions for nonlinear discrete time systems by linear programming. Journal of Difference Equations and Applications, 2014 (in press).

[8] S. P. Gordon. On converses to the stability theorems for difference equations. SIAM J. Control, 10(1):76-81, 1972.

[9] S. Hafstein, C. M. Kellett, and H. Li. Continuous and piecewise affine Lyapunov functions using the Yoshizawa construction. To appear at the 2014 American Control Conference.

[10] W. Hahn. Stability of Motion. Springer-Verlag, 1967.

[11] W. D. Kalies, K. Mischaikow, and R. C. A. M. VanderVorst. An algorithmic approach to chain recurrence. Found. Comput. Math., 5(4):409-449, 2005

[12] C. M. Kellett. A compendium of comparsion function results. Accepted to Mathematics of Control, Signals and Systems, February 2014.

[13] C. M. Kellett and A. R. Teel. Smooth Lyapunov functions and robustness of stability for difference inclusions. Systems and Control Letters, 52(5):395-405, August 2004.

[14] C. M. Kellett and A. R. Teel. On the robustness of $\mathcal{K} \mathcal{L}$-stability for difference inclusions: smooth discrete-time Lyapunov functions. SIAM J. Control Optim., 44(3):777-800 (electronic), 2005.

[15] S. Marinosson. Lyapunov function construction for ordinary differential equations with linear programming. Dynamical Systems, 17:137150, 2002.

[16] E. D. Sontag. Comments on integral variants of ISS. Systems and Control Letters, 34(1-2):93-100, 1998.

[17] A. Stuart and A. Humphries. Dynamical Systems and Numerical Analysis. Cambridge University Press, 1996.

[18] T. Yoshizawa. Stability Theory by Liapunov's Second Method. Mathematical Society of Japan, 1966. 